Review Article

\title{
Proton Pump Inhibitors and Dementia: Physiopathological Mechanisms and Clinical Consequences
}

\author{
Gloria Ortiz-Guerrero, ${ }^{1}$ Diana Amador-Muñoz, ${ }^{2}$ Carlos Alberto Calderón-Ospina, ${ }^{3}$ \\ Daniel López-Fuentes, ${ }^{4}$ and Mauricio Orlando Nava Mesa $\left(^{2}\right.$ \\ ${ }^{1}$ Individualized Research Learner Program, Neuromuscular Research Division, University of Kansas Medical Center, 3901 Rainbow \\ Blvd., Kansas City, KS 66160, USA \\ ${ }^{2}$ Neuroscience (NEUROS) Research Group, School of Medicine and Health Sciences, Universidad del Rosario, Carrera 24 No. 63C-69, \\ Bogotá 111221, Colombia \\ ${ }^{3}$ Unidad de Farmacología, School of Medicine and Health Sciences, Universidad del Rosario, Carrera 24 No. 63C-69, \\ Bogotá 111221, Colombia \\ ${ }^{4}$ Medical Social Service, Hospital de San Francisco, Kra 8 No. 6A-121, Gacheta 251230, Colombia
}

Correspondence should be addressed to Mauricio Orlando Nava Mesa; monavam@usal.es

Received 14 November 2017; Accepted 14 February 2018; Published 21 March 2018

Academic Editor: Francisco Lopera

Copyright (c) 2018 Gloria Ortiz-Guerrero et al. This is an open access article distributed under the Creative Commons Attribution License, which permits unrestricted use, distribution, and reproduction in any medium, provided the original work is properly cited.

\begin{abstract}
Alzheimer's disease (AD) is the most common type of dementia, mainly encompassing cognitive decline in subjects aged $\geq 65$ years. Further, $\mathrm{AD}$ is characterized by selective synaptic and neuronal degeneration, vascular dysfunction, and two histopathological features: extracellular amyloid plaques composed of amyloid beta peptide $(\mathrm{A} \beta)$ and neurofibrillary tangles formed by hyperphosphorylated tau protein. Dementia and AD are chronic neurodegenerative conditions with a complex physiopathology involving both genetic and environmental factors. Recent clinical studies have shown that proton pump inhibitors (PPIs) are associated with risk of dementia, including AD. However, a recent case-control study reported decreased risk of dementia. PPIs are a widely indicated class of drugs for gastric acid-related disorders, although most older adult users are not treated for the correct indication. Although neurological side effects secondary to PPIs are rare, several preclinical reports indicate that PPIs might increase $\mathrm{A} \beta$ levels, interact with tau protein, and affect the neuronal microenvironment through several mechanisms. Considering the controversy between PPI use and dementia risk, as well as both cognitive and neuroprotective effects, the aim of this review is to examine the relationship between PPI use and brain effects from a neurobiological and clinical perspective.
\end{abstract}

\section{Introduction}

Dementia is a clinical syndrome that represents a wide spectrum of cognitive dysfunction and leads to progressive and chronic deterioration of social and occupational activities. According to the World Alzheimer Report, over 46.8 million people worldwide lived with dementia in 2015 , with a predicted increase of cases to 74.7 million by 2030 and 131.5 million by 2050 . In addition, $63 \%$ and $68 \%$ of all people with dementia will live in low- and middle-income countries by 2030 and 2050, respectively [1]. Because of this increased number of cases, the high cost of dementia is another issue that health systems will be dealing with in the future.
Currently, the cost is estimated at $\$ 18$ billion per year in the US, with an increase expected over upcoming years. Owing to the economic and social impact caused by dementia, the World Health Organization designated dementia a public health priority [2]. There are different types of dementia, with Alzheimer's disease (AD) being the most prevalent in humans, accounting for $50-70 \%$ of all cases [3]. The prevalence rate for $\mathrm{AD}$ increases predominantly with age, surging from $3.5 \%$ in people aged 75 years old to $46.3 \%$ in people aged 95 years old or older [4]. The histopathological hallmarks of $\mathrm{AD}$ include extracellular deposition of amyloid- $\beta$ (A $\beta)$ plaques, formation of neurofibrillary tangles (NFTs) from hyperphosphorylated tau protein, and 
neurodegeneration caused by progressive loss of neurons and their processes [5]. Moreover, the most accepted theory to explain the pathogenesis of $\mathrm{AD}$ is the amyloid hypothesis, which states that the cognitive disease phenotype is due to $\mathrm{A} \beta$ dyshomeostasis [6].

Proton pump inhibitors (PPIs) are a class of drugs used to treat gastric acid-related disorders, such as gastroesophageal reflux and peptic ulcer disease, and which act mainly as irreversible inhibitors of the $\mathrm{H}^{+} / \mathrm{K}^{+}$-ATPase pump to decrease gastric acid production [7]. PPIs have an excellent safety profile and have become one of the most prescribed drugs in recent years. According to the National Health and Nutrition Examination Survey, from 1999 to 2012, the percentage of adults aged 40-60 who received a prescription for PPIs almost doubled from $4.9 \%$ to $8.3 \%$ in the United States, surging concerns about their widespread use among this age group $[8,9]$. Furthermore, various studies have shown that $50-70 \%$ of patients prescribed PPIs do not have the correct indication, especially in hospitalized elderly patients [10-12]. Overall, long-term use of PPIs has increased, leading to potential adverse effects such as nutritional deficiencies (vitamin $\mathrm{B}_{12}$, magnesium, and iron), renal damage, osteoporotic fracture, infection by Clostridium difficile, rhabdomyolysis, anemia, and thrombocytopenia [13]. Because of these adverse effects, their safety and role in cognitive function (including risk of developing dementia and $\mathrm{AD}$ ) have been questioned lately. Several studies described association between PPIs and greater risk of developing dementia and $\mathrm{AD}$ in older people $[9,14,15]$. However, other study has not shown that PPIs were associated with greater risk of dementia neither $\mathrm{AD}$ [7]. In addition, a recent casecontrol study conducted in German primary care patients reported decreased risk of dementia with PPI use [16]. In fact, neuroprotective effects of PPIs have been recently described $[17,18]$. Due to these controversial findings, and also the role of PPIs in progression from mild to severe cognitive dysfunction, the aim of this article is to review the relationship between PPI use and basic mechanisms of neuronal dysfunction. In this regard, we discuss if PPI use is associated with greater susceptibility to developing dementia, focusing on a neurobiological basis of $\mathrm{AD}$. Consequently, we propose new hypothesis regarding the physiopathological mechanisms of cognitive impairment induced by acute and chronic PPI use and examine some associated factors that increase dementia susceptibility after PPI exposure.

\section{The Effect of PPIs on the Central Nervous System}

One of the human genes encoding $\mathrm{H}^{+} / \mathrm{K}^{+}$-ATPase (ATP12A/ ATP1AL1) is expressed in the brain, colon, and placenta, while the other gene (ATP4A) is only expressed in gastric epithelial cells [19]. Accordingly, there is evidence of $\mathrm{H}^{+}$/ $\mathrm{K}^{+}$-ATPase activity in the central nervous system (CNS), with certain isoforms expressed [20]. Proton pumps have several physiological functions in neurons and contribute to acid-base and potassium homeostasis [19]. Vesicular proton pumps $\left(\mathrm{H}^{+}\right.$-ATPases or $\mathrm{V}$-ATPases $)$ create the proton gradient that is required for packaging of neurotransmitters into synaptic vesicles. Furthermore, new evidence indicates that vesicular $\mathrm{H}^{+} / \mathrm{K}^{+}$-ATPase plays an interesting role in both exocytosis and endocytosis in nerve terminals [21, 22]. $\mathrm{Ca}^{2+}$-ATPase, $\mathrm{Na}^{+} / \mathrm{K}^{+}$-ATPase, and $\mathrm{H}^{+} / \mathrm{K}^{+}$-ATPase are included in the PII subfamily of P-type ATPases [23]. P-type ATPases share common structural motifs and likely arise from a common ancestral gene [19]. Interestingly, the primary structure of the $\alpha$ subunit of gastric $\mathrm{H}^{+} / \mathrm{K}^{+}$-ATPase is $98 \%$ homologous within species and highly homologous to the catalytic subunit of $\mathrm{Na}^{+} / \mathrm{K}^{+}$-ATPase $(\sim 63 \%)$ and sarcoplasmic/endoplasmic reticulum $\mathrm{Ca}^{2+}$-ATPase (SERCA) $(\sim 25 \%)[24,25]$.

Proton pump inhibitors (e.g., omeprazole, lansoprazole, dexlansoprazole, rabeprazole, pantoprazole, and esomeprazole) effectively block acid secretion by covalent and irreversible binding to $\mathrm{H}^{+} / \mathrm{K}^{+}$-ATPases on the luminal surface of the parietal cell membrane $[26,27]$. The site of reaction on the enzyme differs according to the particular PPI. However, all PPIs react with cysteine 813 in the active E2 configuration (ion-site-out) [27]. Considering the high homology between P-type ATPases, it is possible that PPIs can inhibit other ionic pumps in different organs or even induce systemic physiological changes. Indeed, the CNS may be one system affected, with its interaction facilitated by pathological conditions exhibiting reduced $\mathrm{pH}$ in the brain, cerebrospinal fluid, and blood (i.e., metabolic stress).

Passage of PPIs through the blood-brain barrier (BBB) has been calculated. After administering $10 \mathrm{mg} / \mathrm{kg}$ intravenous (IV) omeprazole to male Sprague Dawley rats, the area under the curve (AUC) of concentration versus time in the brain divided by AUC in blood was calculated [28]. The resulting blood-to-brain distribution coefficient was 0.15 , indicating that up to $15 \%$ of a single IV dose of omeprazole can reach the CNS and potentially affect cognitive function with either acute or repetitive long-term use. Corroboratively, in vitro and in vivo pharmacokinetic studies have shown that lansoprazole may also penetrate the BBB [29].

Some PPIs, such as lansoprazole, esomeprazole, and pantoprazole, are reported to cause adverse neurological effects, mainly headaches [30,31] and dizziness/vertigo [32]. Other adverse effects that involve the CNS (at a frequency of $<1 \%$ ) include depression, diplopia, disturbed sleep, drowsiness, insomnia, nervousness, and tremor. There have also been reports of sensoperceptual abnormalities (i.e., hallucinations) $[33,34]$ and delirium [35]. Neurological side effects induced by chronic PPI use may be related to indirect systemic abnormalities (i.e., magnesium and vitamin $B_{12}$ deficiency) [36] or direct effects on neurons after passage through the BBB. Although the exact mechanisms on brain circuits have not been fully described, most neurological side effects are reported with chronic administration of PPIs.

\section{PPIs and Physiopathological Effects in Dementia}

PPI drugs can facilitate tau and $\mathrm{A} \beta$-induced neurotoxicity, which may increase AD progression and cognitive decline. Below, we discuss relevant physiopathological mechanisms. 
3.1. PPIs and $A \beta$ Plaques. One of the most described effects in dementia relates to increased production of $A \beta$ plaques by PPIs [37]. As already stated, one of the major hallmarks of $\mathrm{AD}$ is extracellular accumulation of $\mathrm{A} \beta$ plaques, which lead to oxidative and inflammatory damage in the brain [3]. These $\mathrm{A} \beta$ species are produced by cleavage of amyloid precursor protein (APP) by $\beta$-secretase (also known as $\beta$-site APPcleaving enzyme 1 [BACE1]) and $\gamma$-secretase [38]. Although the total number of $A \beta$ plaques does not correlate well with $\mathrm{AD}$ severity, there is a direct effect on cognition and cell death in APP/tau transgenic mice because of neuronal loss and the astrocyte inflammatory response [39]. In 2013, Badiola et al. [37] investigated the effect of PPIs on $\mathrm{A} \beta$ production using cell and animal models and suggested a novel hypothesis: they suggested that PPIs act as inverse $\gamma$-secretase modulators (iGSM), which change the $\gamma$-secretase cleavage site and thereby increase $A \beta 42$ levels and decrease $A \beta 38$ levels. In addition, PPIs can increase BACE1 activity, raising production of $A \beta 37$ and $A \beta 40$ levels. In $A D$, the major pathological species is thought to be $\mathrm{A} \beta 42$, but the most produced is $\mathrm{A} \beta 40$ [38]. Ultimately, PPIs (specifically lansoprazole) may alter media $\mathrm{pH}$, amplifying the activity of other proteases, such as memprin- $\beta$, and generating $A \beta 2$-x peptides (e.g., $\mathrm{A} \beta 2-37, \mathrm{~A} \beta 2-40$, and $\mathrm{A} \beta 2-42$ species). Moreover, Badiola et al. were able to demonstrate that lansoprazole enhances $\mathrm{A} \beta$ production using in vivo and in vitro models, supporting the theory that PPIs effect $\mathrm{AD}$ by boosting $\mathrm{A} \beta$ production [37]. It has also been shown that PPIs can inhibit vacuolar proton pumps, which acidify lysosomes by pumping protons from the cytoplasm to the lumen of vacuoles in microglia and macrophages $[40,41]$. Normally, this acid environment in lysosomes permits degradation of fibrillary $\mathrm{A} \beta$. As PPIs can cross the BBB, they act on V-ATPases in an inhibitory manner, causing less degradation of fibrillary $\mathrm{A} \beta$ and hence a reduction in its clearance $[28,41]$. To date, there are few studies that explain the relationship between the effects of PPIs and presence of $\mathrm{A} \beta$ plaques. It would be interesting if future studies determine why $\mathrm{A} \beta$ plaque production increases or their clearance decreases with PPI use.

3.2. PPIs and Tau Protein. Currently, $\mathrm{AD}$ diagnosis is based on neuropsychological tests (cognitive criteria), neuroimaging (i.e., MRI and amyloid deposits by PET), and tau/amyloid in CSF (biomarker criteria) that rule out other causes of dementia [42-44]. However, a definitive diagnosis can only be confirmed histopathologically by the extensive presence of $\mathrm{A} \beta$ and NFTs in the neocortex of postmortem brain tissue [45]. The main component of NFTs is paired helical filaments (PHFs) formed from hyperphosphorylated tau protein $[46,47]$. Tau protein plays an important role as a microtubule-associated protein in neuronal axons, stabilizing microtubules and inducing their assembly [48]. When tau protein is hyperphosphorylated, it is unable to bind and stabilize microtubules, which leads to degeneration of affected neurons [49]. According to the neuroimmunomodulation theory of $\mathrm{AD}$, the earliest $\mathrm{CNS}$ changes before the clinical onset of $\mathrm{AD}$ result from a chronic inflammatory response, which leads to abnormal tau phosphorylation and induces formation of PHFs and tau protein aggregates, ultimately resulting in cytoskeletal alterations [50]. Consequently, these lesions are present before the presentation of clinical symptoms of $\mathrm{AD}$ [51]. The first NFT lesion appears in the transentorhinal cortex and is proceeded by the entorhinal cortex and hippocampus and finally the neocortex [52]. Several studies have shown that NFTs correlate with cognitive decline and severity in $\mathrm{AD}$, positioning tau NFTs as suitable targets for therapy and diagnosis in AD patients. Several researchers have focused on developing different radiotracer components based on their high affinity for tau protein. This will allow future quantification of tau burden using noninvasive diagnostic imaging such as positron emission tomography (PET) [53]. Okamura and coworkers have found that PPIs show high affinity to tau protein. They screened more than 2000 compounds to develop agents for use in PET and identified quinoline and benzimidazole as high affinity components of NFTs rather than senile plaques [51]. Subsequently, Rojo and colleagues found that lansoprazole, a Food and Drug Administration- (FDA-) approved PPI with a benzimidazole ring structure, had nanomolar binding affinity for tau aggregates, in agreement with Okamura's findings. They also showed that lansoprazole has high lipophilicity and can cross the BBB, reaching the brain within $37 \mathrm{~min}$ after administration and therefore showing suitability as a radiotracer for PET imaging. However, during kinetic analysis, lansoprazole interactions with tau NFTs did not fit the classical one-site or two-site binding models [29]. We hypothesize that this can be explained by the six tau protein isoforms expressed in the human CNS. These tau isoforms are divided into two sets with three (3R) and four (4R) microtubulebinding domains. In usual conditions, both sets of isoforms are expressed in an equal ratio, while under pathological conditions, different tauopathies show different isoform ratios with diverse morphologies [54]. Additionally, tau undergoes multiple posttranslational changes resulting in conformational modifications in aggregates, as well as alterations in binding affinities and binding sites of tau protein [55]. Further, Rojo et al. performed docking studies and identified strong hydrogen bond interactions between the $\mathrm{NH}$ group of the benzimidazole ring of lansoprazole and the C-terminal hexapeptide (386TDHGAE391) of the tau core [29]. Corroboratively, studies by Fawaz and coworkers determined that replacing the $\mathrm{NH}$ group of the benzimidazole ring affected tau protein affinity, which has enabled development of new radiotracers with higher affinities, such as $[18 \mathrm{~F}] \mathrm{N}$-methyl lansoprazole [53]. This research field is based on the study of lansoprazole, and indeed its high affinity to tau protein is a striking and open avenue for researchers to create and improve noninvasive techniques for diagnosing $\mathrm{AD}$ in the early stages. Many of these studies are still in preclinical or early clinical trial stages. Further investigations are needed to specifically understand PPI interactions with tau protein.

3.3. PPIs and Vitamin $B_{12}$ Deficiency. Gastric acidity is necessary for absorption of vitamin $B_{12}$, which is an essential water-soluble vitamin obtained from different dietary sources such as fish, meat, dairy products, and fortified cereal [56]. Statistically, approximately 6-20\% of American adults have vitamin $B_{12}$ deficiency, likely as a natural phenomenon. 
Nonetheless, the risk of $\mathrm{B}_{12}$ deficiency increases with age [57]. During digestion, vitamin $B_{12}$ binds to salivary $R$ proteins and then to intrinsic factors, reaching the whole intestine and ileum terminal intact, where $\mathrm{B}_{12}$ absorption occurs. $B_{12}$ is firmly bound to proteins and consequently requires acid-activated proteolytic digestion. Use of PPIs causes hypochlorhydria, which results in vitamin $B_{12}$ malabsorption as $B_{12}$ remains tightly bound to proteins in the stomach [58]. Several studies have shown controversial results between long-term PPI use and vitamin $B_{12}$ deficiency. For instance, in a case-control study, patients treated with PPIs for 2 years or longer showed a statistically significant association with increased risk of $B_{12}$ deficiency [59]. In contrast, in a cross-sectional study, patients prescribed PPIs for 3 years or longer had similar $B_{12}$ levels as non-PPI users [60]. It is important to note that most of these trials show association and not causation. Likely, other factors will contribute to these findings in addition to acid-suppressive therapy [61].

Vitamin $B_{12}$ deficiency caused by PPI use has been associated with dementia and cognitive impairment [62]. Vitamin $B_{12}$ is required for one-carbon transfer reactions such as methylation, which are needed for processing and production of nucleotides, phospholipids, and monoamine neurotransmitters [63]. Usually, vitamin $B_{12}$ removes a methyl group from tetrahydrofolate, turning it into methylcobalamin. Methylcobalamin then presents its methyl group to homocysteine, which is finally converted to methionine by methionine synthase [62]. Thus, vitamin $B_{12}$ deficiency is one of the main causes of hyperhomocysteinemia. Both hyperhomocysteinemia and $\mathrm{B}_{12}$ deficiency are considered risk factors for brain atrophy, cognitive impairment, and dementia [64]. Studies have shown that hyperhomocysteinemia may activate several protein kinases, such as glycogen synthase kinase $3 \beta$ (GSK-3 $\beta$ ), cyclin-dependent kinase-5 (Cdk-5), c-Jun $\mathrm{N}$-terminal kinase (JNK), extracellular signal-regulated kinase (ERK), and p38 mitogen-activated protein kinase (MAPK), and inhibit protein phosphatase 2A (PP2A) (41), which are all pivotal enzymes in regulating the phosphorylation state of tau protein [65]. Among these enzymes, PP2A plays a crucial role as it is the main brain serine/threonine phosphatase and prevents tau hyperphosphorylation [66]. Decreased methylation may affect PP2A function by leading to hyperphosphorylation and aggregation of tau protein [62]. In animal models, aside from inducing tau protein hyperphosphorylation, hyperhomocysteinemia can increase $\mathrm{A} \beta$ production, while folate/vitamin $B_{12}$ supplementation may attenuate these effects [67-69]. Based on these findings, high homocysteine levels are a strong and independent risk factor for developing $\mathrm{AD}$ [70]. Alternative mechanisms for linking $\mathrm{AD}$ with vitamin $\mathrm{B}_{12}$ deficiency have also been described, which are distinct from PP2A inactivation and tau hyperphosphorylation. Using the structure and function of vitamin $B_{12}$ inside cells, Rafiee and colleagues (38) [62] studied direct binding between $B_{12}$ and tau protein in vitro by fluorometry and circular dichroism. Because vitamin $B_{12}$ can interact with thiol groups, they determined that cobalamin can directly bind to tau via cysteine residues on tau protein. Hence, the resulting $B_{12} /$ tau protein complex prevents tau protein fibrillation. Besides, tau aggregation is inhibited by vitamin $B_{12}$ capping on cysteine residues of tau. In summary, the effect exerted on neurodegeneration by vitamin $\mathrm{B}_{12}$ deficiency and hyperhomocysteinemia is not only due to PP2A inactivation and tau hyperphosphorylation but also direct binding of vitamin $\mathrm{B}_{12}$ to tau protein, inhibiting its fibrillation and aggregation [62].

Although different mechanisms have been described to explain the effects of vitamin $B_{12}$ deficiency in dementia, more clinical trials are needed to understand this relationship. In addition, more studies are required to establish if vitamin $B_{12}$ deficiency is a causal event in dementia or an associated factor, as shown in other studies.

\section{PPIs and Antineurotoxicity}

In contrast to the effect of PPIs on $A \beta$ plaque production, tau protein, and vitamin $\mathrm{B}_{12}$ deficiency in $\mathrm{AD}$ development, different studies have shown antineurotoxic effects of PPIs on astrocytes and microglia [17]. In $\mathrm{AD}$ patients, activated astrocytes exist in close relationship with senile plaques and neuronal degeneration [71]. These activated cells release powerful neurotoxic products, including proinflammatory cytokines, reactive oxygen species, and nitric oxide [72, 73]. Although astrocytes show neuroprotective activity, activated astrocytes may aggravate neurodegenerative diseases [74]. Hashioka et al. [17] demonstrated that lansoprazole and omeprazole decrease interferon- (IFN-) $\gamma$-induced astrocytic neurotoxicity as a result of inhibition of signal transducer and activator of transcription 3 (STAT3) activation. They also confirmed that PPIs slightly reduce production of T-cell alpha chemoattractant (I-TAC) by IFN- $\gamma$-activated astrocytes, although only lansoprazole shows a significant effect. These findings are in agreement with another study by Hashioka et al. [18] showing that lansoprazole and omeprazole may suppress tumor necrosis factor- (TNF-) $\alpha$ production from THP-1 cells and decrease human microglial and monocyte neurotoxicity. In addition, they showed that nonsteroidal anti-inflammatory drugs (NSAID) can increase antineurotoxic effect of PPIs due to their synergic effect. Furthermore, a recent study identified lansoprazole as a liver $\mathrm{X}$ receptor (LXR) agonist [75]. LXRs are transcription factors that modify expression of genes related to cholesterol metabolism. Hence, lansoprazole increases ATP-binding cassette transporter (Abca1) and apolipoprotein- (Apo-) E levels in primary astrocytes, both genes regulated by LXR [75]. Moreover, ApoE lipid complexes mediated by ABCA1 inhibit $\mathrm{A} \beta$ plaque aggregation, thereby supporting the theory that lansoprazole can act as a therapeutic agent in $\mathrm{AD}$ [17].

\section{Clinical Association between PPIs and Risk of Dementia, AD, and Cognitive Impairment}

Based on a systematic review from 2017, four European observational studies have investigated association between PPI use and dementia. Three studies have found a positive association between dementia and omeprazole, esomeprazole, lansoprazole, and pantropazole, with an approximately 1.4-fold increased risk of any dementia in cohorts using PPIs 
(95\% CI, 1.36-1.52; $P<0.001$ ) [76]. Similarly, in a recent prospective cohort study in Asian population $(n=15726$, 7863 PPI users), an association with dementia has been found (aHR, 1.22; 95\% confidence interval, 1.05-1.42) [15]. In contrast, the fourth European report included in the systematic review found a negative association (OR dementia with PPI use $=0.94$ (95\% CI, 0.90-0.97) $P=0.0008$ ) [76]. Herghelegiu and colleagues [77] conducted a single-center case-control study that compared 148 PPI users (aged at least 65 years old) with a control group of nonusers during an 8 -month period. They confirmed statistically significant association between PPI use and dementia. However, bias was influenced by the small study sample. Further, they did not take into account fundamental cofounders such as age, sex, history of stroke, and smoking status, which are considered risk factors for dementia. Conversely, Booker and coworkers [16] found statistically significant reduction between dementia and PPI use with a case-control study using a database of general practice medical records in Germany and including 11,956 patients with initial dementia diagnoses over a 4 -year period. This study was considered to have a moderate risk of bias due to codes being entered by general practitioners. The other two cohort studies performed by Haenisch et al. and Gomm et al. $[9,14]$ used data from two different German databases (KNDD and AgeCoDe, resp.) which reported positive association between PPI use and dementia. Although both studies included a wide range of cofounders, neither took into account either hypertension or family history of dementia (again well-known risk factors for dementia) nor sugar intake, physical exercise, or air pollution, recently considered risk factors for dementia [76]. In contrast, Haenisch et al. [14] performed the only study to include a subgroup for evaluating AD risk. Their results were in favor of elevated risk for $\mathrm{AD}$ associated with PPI use. Besides, exclusion of cofounders (such as age, sex, education, ApoE4 allele status, polypharmacy, depression, ischemic heart disease, and stroke) did not influence the effect of PPI use on AD.

In the same systematic review, weak association between PPI use and acute cognitive impairment was demonstrated in a series of case reports and small observational studies [76]. Regarding these, four were hypomagnesemia-associated delirium or confusion cases $[78,79]$, one was associated with hyponatremic delirium [80], and one was delirium of unknown cause [35]. PPIs were implied as a main cause in these effects because they can lower magnesium and sodium levels, and the majority of studies were associated with omeprazole use $[35,78,80]$. In one case, withdrawing esomeprazole therapy provided symptomatic relief and reestablishment of magnesium levels [78]. With these observational studies, data on PPI use and risk of delirium was incorporated [81, 82]. For instance, Otremba and coworkers performed a cross-sectional study, including 675 patients of 60-100 years old, who were admitted to a subacute geriatric ward over a 12 -month period. They found that PPI use was a predictive factor for developing delirium in these patients. The Confusion Assessment Method and Delirium-O-Meter were the scales used for diagnosing delirium and its severity, respectively [82]. However, Fujii and coworkers, who compared incidence and severity of delirium in $\mathrm{H}(2)$ blocker users and PPI users, found that delirium can be reduced by switching $\mathrm{H}(2)$ blockers to PPIs [81]. However, there are major difficulties in evaluating these studies as the data does not contain reliable information. Also, the majority of studies used different group characteristics, which makes comparisons among them challenging [76].

Other studies have focused on short-term PPI use and its influence on different cognitive functions. For instance, Akter et al. used the Cambridge Neuropsychological Test Automated Battery (the well-known CANTAB software) to evaluate each PPI and its effect in different cognitive domain functions of young patients (20-26 years old) over 7 days [12]. CANTAB software can accurately determine amyloidrelated cognitive decline and quantify the severity of impairment in prodromal $\mathrm{AD}$ patients [83]. This study found that taking different PPIs may influence several degrees of cognitive capacity. For instance, omeprazole led to deterioration of visual and episodic memory, motor and mental response speed, new learning, short-term and sustained attention, retention and manipulation of visuospatial information, and strategy development [12]. Lansoprazole, in addition to the mentioned effects of omeprazole, can restrict manipulation of remembered memory to generate a complex task or strategy and also limit retention of spatial information. Contrary to lansoprazole and omeprazole, esomeprazole induced difficulties in maintaining sustained attention, retaining and manipulating spatial memory, and planning strategy [12]. In contrast, a very recent study including data from the Nurses' Health Study II has not shown association between PPI use and cognitive dysfunction or dementia risk. However, this study indicates a modest association for psychomotor speed and attention among PPI users [84]. To explain some acute and chronic cognitive effects, it is possible that PPIs may preferentially affect the hippocampus and associative neocortex via a neuroplasticity mechanism. Still to our knowledge, there is no evidence of in vivo or in vitro experiments using long-term potentiation protocols to confirm this theory.

\section{Final Considerations and Conclusion}

There is currently no consensus on the role of PPIs and the associated risk of developing dementia. Because of the multifactorial origin of dementia (Alzheimer-vascular spectrum dementia), future studies are required to consider associated environmental and genetic factors, as well as biomarkers (i.e., APOE- $\varepsilon 4$ ) and other covariates (i.e., chronic stress) that may increase the risk of dementia in patients who consume PPIs. It is possible that the cognitive effects of PPIs are due to drug interactions, especially in polymedicated elderly patients. For instance, omeprazole may increase blood levels of diazepam (a $\gamma$-aminobutyric acid [GABA]-A agonist) by decreasing plasma clearance (via cytochrome P450) and then increasing neurological side effects [85]. In a retrospective study in six residential care homes in England $(n=133)$, it was found that 9.2\% of older people with dementia were prescribed with two or more 
potentially inappropriate medications, including PPIs and long-acting benzodiazepines [86]. In addition, an FDA study has shown that some adverse events with PPIs (including omeprazole, lansoprazole, and pantoprazole) could be associated with benzodiazepine drug interactions [87]. As long-term benzodiazepine use might increase dementia risk [88,89], it is possible that combined treatment of GABA-A agonists and PPIs may increase this susceptibility, considering the close association between GABAergic system dysfunction and the physiopathology of AD and mild cognitive impairment [90]. In fact, cognitive impairment has been reported after lorazepam treatment in patients with higher risk for $\mathrm{AD}$ (APOE- 44 allele carriers) [91]. At recommended doses, a pharmacokinetic interaction between benzodiazepines and PPIs is less probable with pantoprazole, lansoprazole, and rabeprazole than with omeprazole [92-94]. Accordingly, those PPI drugs might be considered in polymedicated subjects.

Cumulative evidence indicates that chronic treatment with NSAIDs (e.g., ibuprofen) may delay $\mathrm{AD}$ onset and reduce $\mathrm{AD}$ rate of progression $[95,96]$. As mentioned earlier, in vitro evidence indicates that PPIs might have neuroprotective and anti-inflammatory effects that act synergistically with NSAIDs. Some limitations of in vitro studies include the experimental duration, as well as complex specific microenvironment factors observed only in vivo [97]. In fact, dementia and $\mathrm{AD}$ are chronic neurodegenerative diseases with a complex physiopathology and several compensatory neuronal-glial mechanisms after long-term $\mathrm{A} \beta$ peptide exposure. To simulate environmental cellular conditions of $\mathrm{AD}$, it is necessary to design durable in vitro studies that involve persistent oxidative stress and metabolic dysfunction.

Vascular and BBB dysfunctions have been observed in AD patients [98]. Taking into account age-related changes of $\mathrm{BBB}$ function, as well as vulnerability to disruption by external factors such as hypertension and drugs [99, 100], we suggest that neurological susceptibility to PPIs may be related to changes in BBB permeability, as well as changes in the brain microenvironment related to aging. However, more studies are necessary to identify other factors that contribute to this susceptibility.

Although the mechanisms of brain dysfunction induced by PPIs are not known with certainty, it is possible they influence ionic pumps controlling the membrane potential and electrochemical gradient in neurons. Considering the preferential effects of PPIs on $\mathrm{A} \beta$ and tau protein, as well as on endothelial function, further studies are needed to contemplate differential susceptibility between $\mathrm{AD}$ and vascular dementia. Taking into account the effects of PPIs on vitamin $B_{12}$ levels, and possibly indirect effects on membrane ionic transporters, nutritional and electrolyte monitoring is required in patients who chronically use PPIs, mainly older adults and patients with chronic malnutrition or debilitating chronic conditions. Also, it is necessary to determine the previous cognitive status of patients and whether they have risk factors for dementia, as well as pharmacokinetic drug interactions. Altogether, it is necessary to consider the riskbenefit of chronic PPI use and, above all, strictly establish an adequate therapeutic indication.

\section{Conflicts of Interest}

The authors declare that they have no conflicts of interest.

\section{Acknowledgments}

The authors thank Rachel James, Ph.D., from Edanz Group (http://www.edanzediting.com/ac) for editing a draft of this manuscript. This work was supported by the Universidad del Rosario (Bogotá, Colombia).

\section{References}

[1] M. Prince, A. Wimo, M. Guerchet, G. Claire Ali, Y.-T. Wu, and M. Prina, World Alzheimer Report 2015: the Global Impact of Dementia: an Analysis of Prevalence, Incidence, Cost and Trends, Alzheimer's Disease International (ADI), London, 2015.

[2] World Health Organization, Dementia: a Public Health Priority. World Health Organization, World Health Organization, Geneva, 2012.

[3] H. W. Querfurth and F. M. LaFerla, "Alzheimer's disease," The New England Journal of Medicine, vol. 362, no. 4, pp. 329-344, 2010.

[4] N. Matyas, S. Auer, C. Gisinger et al., "Continuing education for the prevention of mild cognitive impairment and Alzheimer's-type dementia: a systematic review protocol," Systematic Reviews, vol. 6, no. 1, p. 157, 2017.

[5] P. S. Aisen, J. Cummings, C. R. Jack et al., "On the path to 2025: understanding the Alzheimer's disease continuum," Alzheimer's Research \& Therapy, vol. 9, no. 1, p. 60, 2017.

[6] D. J. Selkoe and J. Hardy, "The amyloid hypothesis of Alzheimer's disease at 25 years," EMBO Molecular Medicine, vol. 8, no. 6, pp. 595-608, 2016.

[7] F. C. Goldstein, K. Steenland, L. Zhao, W. Wharton, A. I. Levey, and I. Hajjar, "Proton pump inhibitors and risk of mild cognitive impairment and dementia," Journal of the American Geriatrics Society, vol. 65, no. 9, pp. 19691974, 2017.

[8] E. D. Kantor, C. D. Rehm, J. S. Haas, A. T. Chan, and E. L. Giovannucci, "Trends in prescription drug use among adults in the United States from 1999-2012," JAMA, vol. 314, no. 17, pp. 1818-1831, 2015.

[9] W. Gomm, K. von Holt, F. Thomé et al., “Association of proton pump inhibitors with risk of dementia: a pharmacoepidemiological claims data analysis," JAMA Neurology, vol. 73, no. 4, pp. 410-416, 2016.

[10] J. J. Heidelbaugh and J. M. Inadomi, "Magnitude and economic impact of inappropriate use of stress ulcer prophylaxis in non-ICU hospitalized patients," The American Journal of Gastroenterology, vol. 101, no. 10, pp. 2200-2205, 2006.

[11] S. M. Eid, A. Boueiz, S. Paranji, C. Mativo, R. Landis, and M. S. Abougergi, "Patterns and predictors of proton pump inhibitor overuse among academic and non-academic hospitalists," Internal Medicine, vol. 49, no. 23, pp. 25612568, 2010.

[12] S. Akter, M. R. Hassan, M. Shahriar, N. Akter, M. G. Abbas, and M. A. Bhuiyan, "Cognitive impact after short-term exposure to different proton pump inhibitors: assessment using CANTAB software," Alzheimer's Research \& Therapy, vol. 7, no. 1, p. 79, 2015 
[13] L. Y. Yu, L. N. Sun, X. H. Zhang et al., "A review of the novel application and potential adverse effects of proton pump inhibitors," Advances in Therapy, vol. 34, no. 5, pp. 10701086, 2017.

[14] B. Haenisch, K. von Holt, B. Wiese et al., "Risk of dementia in elderly patients with the use of proton pump inhibitors," European Archives of Psychiatry and Clinical Neuroscience, vol. 265, no. 5, pp. 419-428, 2015.

[15] S.-Y. Tai, C.-Y. Chien, D.-C. Wu et al., "Risk of dementia from proton pump inhibitor use in Asian population: a nationwide cohort study in Taiwan," PLoS One, vol. 12, no. 2, article e0171006, 2017.

[16] A. Booker, L. E. Jacob, M. Rapp, J. Bohlken, and K. Kostev, "Risk factors for dementia diagnosis in German primary care practices," International Psychogeriatrics, vol. 28, no. 7, pp. 1059-1065, 2016.

[17] S. Hashioka, A. Klegeris, and P. L. McGeer, "Proton pump inhibitors reduce interferon- $\gamma$-induced neurotoxicity and STAT3 phosphorylation of human astrocytes," Glia, vol. 59, no. 5, pp. 833-840, 2011.

[18] S. Hashioka, A. Klegeris, and P. L. McGeer, "Proton pump inhibitors exert anti-inflammatory effects and decrease human microglial and monocytic THP-1 cell neurotoxicity," Experimental Neurology, vol. 217, no. 1, pp. 177-183, 2009.

[19] I. R. Driel and J. M. Callaghan, "Proton and potassium transport by $\mathrm{H}^{+} / \mathrm{K}^{+}$-ATPases," Clinical and Experimental Pharmacology and Physiology, vol. 22, no. 12, pp. 952-960, 1995.

[20] N. N. Modyanov, K. E. Petrukhin, V. E. Sverdlov et al., “The family of human $\mathrm{Na}$, K-ATPase genes. ATP1AL1 gene is transcriptionally competent and probably encodes the related ion transport ATPase," FEBS Letters, vol. 278, no. 1, pp. 91-94, 1991.

[21] D. Wang and P. R. Hiesinger, "The vesicular ATPase: a missing link between acidification and exocytosis," The Journal of Cell Biology, vol. 203, no. 2, pp. 171-173, 2013.

[22] L. Tabares and B. Betz, "Multiple functions of the vesicular proton pump in nerve terminals," Neuron, vol. 68, no. 6, pp. 1020-1022, 2010.

[23] M. Bublitz, H. Poulsen, J. P. Morth, and P. Nissen, "In and out of the cation pumps: P-type ATPase structure revisited," Current Opinion in Structural Biology, vol. 20, no. 4, pp. 431439, 2010.

[24] K. J. Sweadner and C. Donnet, "Structural similarities of Na, $\mathrm{K}$-ATPase and SERCA, the Ca2+-ATPase of the sarcoplasmic reticulum," The Biochemical Journal, vol. 356, no. 3, pp. 685704, 2001.

[25] F. Jaisser, J. D. Horisberger, K. Geering, and B. C. Rossier, "Mechanisms of urinary $\mathrm{K}+$ and $\mathrm{H}+$ excretion: primary structure and functional expression of a novel H, K-ATPase," The Journal of Cell Biology, vol. 123, no. 6, pp. 14211429, 1993.

[26] J. M. Shin and N. Kim, "Pharmacokinetics and pharmacodynamics of the proton pump inhibitors," Journal of Neurogastroenterology and Motility, vol. 19, no. 1, pp. 25-35, 2013.

[27] J. M. Shin, K. Munson, O. Vagin, and G. Sachs, "The gastric HK-ATPase: structure, function, and inhibition," Pflugers Archiv: European Journal of Physiology, vol. 457, no. 3, pp. 609-622, 2009.

[28] F. C. Cheng, Y. F. Ho, L. C. Hung, C. F. Chen, and T. H. Tsai, "Determination and pharmacokinetic profile of omeprazole in rat blood, brain and bile by microdialysis and high-performance liquid chromatography," Journal of Chromatography A, vol. 949, no. 1-2, pp. 35-42, 2002.

[29] L. E. Rojo, J. Alzate-Morales, I. N. Saavedra, P. Davies, and R. B. Maccioni, "Selective interaction of lansoprazole and astemizole with tau polymers: potential new clinical use in diagnosis of Alzheimer's disease," Journal of Alzheimer's Disease, vol. 19, no. 2, pp. 573-589, 2010.

[30] J.-F. Liang, Y.-T. Chen, J.-L. Fuh et al., "Proton pump inhibitor-related headaches: a nationwide population-based case-crossover study in Taiwan," Cephalalgia, vol. 35, no. 3, pp. 203-210, 2014.

[31] R. M. Martin, N. R. Dunn, S. Freemantle, and S. Shakir, "The rates of common adverse events reported during treatment with proton pump inhibitors used in general practice in England: cohort studies," British Journal of Clinical Pharmacology, vol. 50, no. 4, pp. 366-372, 2000.

[32] S. Chimirri, R. Aiello, C. Mazzitello et al., "Vertigo/dizziness as a drugs' adverse reaction," Journal of Pharmacology \& Pharmacotherapeutics, vol. 4, no. 5, pp. 104-109, 2013.

[33] J. J. Sebastian Domingo, "Omeprazole-induced hallucinations. Not as rare as you might think," Gastroenterología y Hepatología, vol. 41, no. 3, 2017.

[34] A. M. Hanneken, N. Babai, and W. B. Thoreson, "Oral proton pump inhibitors disrupt horizontal cell-cone feedback and enhance visual hallucinations in macular degeneration patients," Investigative Ophthalmology \& Visual Science, vol. 54, no. 2, pp. 1485-1489, 2013.

[35] J. G. Heckmann, F. Birklein, and B. Neundörfer, "Omeprazole-induced delirium," Journal of Neurology, vol. 247, no. 1, pp. 56-57, 2000.

[36] C. de la Coba Ortiz, F. Argüelles Arias, C. Martín de Argila de Prados et al., "Proton-pump inhibitors adverse effects: a review of the evidence and position statement by the Sociedad Española de Patología Digestiva," Revista Española de Enfermedades Digestivas, vol. 108, no. 4, pp. 207-224, 2016.

[37] N. Badiola, V. Alcalde, A. Pujol et al., "The proton-pump inhibitor lansoprazole enhances amyloid beta production," PLoS One, vol. 8, no. 3, article e58837, 2013.

[38] S. G. Younkin, "The role of A $\beta 42$ in Alzheimer's disease," Journal of Physiology-Paris, vol. 92, no. 3-4, pp. 289292, 1998.

[39] B. DaRocha-Souto, T. C. Scotton, M. Coma et al., "Brain oligomeric $\beta$-amyloid but not total amyloid plaque burden correlates with neuronal loss and astrocyte inflammatory response in amyloid precursor protein/tau transgenic mice," Journal of Neuropathology \& Experimental Neurology, vol. 70, no. 5, pp. 360-376, 2011.

[40] M. K. Fallahzadeh, A. Borhani Haghighi, and M. R. Namazi, "Proton pump inhibitors: predisposers to Alzheimer disease?," Journal of Clinical Pharmacy and Therapeutics, vol. 35, no. 2, pp. 125-126, 2010.

[41] M. R. Namazi and F. Jowkar, "A succinct review of the general and immunological pharmacologic effects of proton pump inhibitors," Journal of Clinical Pharmacy and Therapeutics, vol. 33, no. 3, pp. 215-217, 2008.

[42] P. J. Visser, S. Vos, I. van Rossum, and P. Scheltens, "Comparison of International Working Group criteria and National Institute on Aging-Alzheimer's Association criteria for Alzheimer's disease," Alzheimer's \& Dementia, vol. 8, no. 6 , pp. 560-563, 2012. 
[43] G. M. McKhann, D. S. Knopman, H. Chertkow et al., "The diagnosis of dementia due to Alzheimer's disease: recommendations from the National Institute on AgingAlzheimer's Association workgroups on diagnostic guidelines for Alzheimer's disease," Alzheimer's \& Dementia, vol. 7, no. 3, pp. 263-269, 2011.

[44] M. C. Carrillo, R. A. Dean, F. Nicolas et al., "Revisiting the framework of the National Institute on Aging-Alzheimer's Association diagnostic criteria," Alzheimer's \& Dementia, vol. 9, no. 5, pp. 594-601, 2013.

[45] D. Dawbarn and S. J. Allen, Neurobiology of Alzheimer's Disease, Bios Scientific, Oxford, 1995.

[46] G. A. Farías, C. Vial, and R. B. Maccioni, "Specific macromolecular interactions between tau and the microtubule system," Molecular and Cellular Biochemistry, vol. 112, no. 1, pp. 8188, 1992.

[47] K. S. Kosik, C. L. Joachim, and D. J. Selkoe, "Microtubuleassociated protein tau (tau) is a major antigenic component of paired helical filaments in Alzheimer disease," Proceedings of the National Academy of Sciences of the United States of America, vol. 83, no. 11, pp. 4044-4048, 1986.

[48] R. B. Maccioni and V. Cambiazo, "Role of microtubuleassociated proteins in the control of microtubule assembly," Physiological Reviews, vol. 75, no. 4, pp. 835-864, 1995.

[49] M. L. Billingsley and R. L. Kincaid, "Regulated phosphorylation and dephosphorylation of tau protein: effects on microtubule interaction, intracellular trafficking and neurodegeneration," The Biochemical Journal, vol. 323, no. 3, Part 3, pp. 577-591, 1997.

[50] L. E. Rojo, J. A. Fernández, A. A. Maccioni, J. M. Jimenez, and R. B. Maccioni, "Neuroinflammation: implications for the pathogenesis and molecular diagnosis of Alzheimer's disease," Archives of Medical Research, vol. 39, no. 1, pp. 1-16, 2008.

[51] N. Okamura, T. Suemoto, S. Furumoto et al., "Quinoline and benzimidazole derivatives: candidate probes for in vivo imaging of tau pathology in Alzheimer's disease," The Journal of Neuroscience, vol. 25, no. 47, pp. 10857-10862, 2005.

[52] H. Braak and E. Braak, "Neuropathological stageing of Alzheimer-related changes," Acta Neuropathologica, vol. 82, no. 4, pp. 239-259, 1991.

[53] M. V. Fawaz, A. F. Brooks, M. E. Rodnick et al., "High affinity radiopharmaceuticals based upon lansoprazole for PET imaging of aggregated tau in Alzheimer's disease and progressive supranuclear palsy: synthesis, preclinical evaluation, and lead selection," ACS Chemical Neuroscience, vol. 5, no. 8, pp. 718-730, 2014.

[54] V. L. Villemagne and N. Okamura, "Tau imaging in the study of ageing, Alzheimer's disease, and other neurodegenerative conditions," Current Opinion in Neurobiology, vol. 36, pp. $43-51,2016$.

[55] M. G. Spillantini and M. Goedert, "Tau pathology and neurodegeneration," The Lancet Neurology, vol. 12, no. 6, pp. 609-622, 2013.

[56] R. Oh and D. L. Brown, "Vitamin $\mathrm{B}_{12}$ deficiency," American Family Physician, vol. 67, no. 5, pp. 979-986, 2003.

[57] A. Hunt, D. Harrington, and S. Robinson, "Vitamin $B_{12}$ deficiency," BMJ, vol. 349, article g5226, 2014.

[58] V. Savarino, P. Dulbecco, and E. Savarino, "Are proton pump inhibitors really so dangerous?," Digestive and Liver Disease, vol. 48, no. 8, pp. 851-859, 2016.
[59] J. R. Lam, J. L. Schneider, W. Zhao, and D. A. Corley, "Proton pump inhibitor and histamine 2 receptor antagonist use and vitamin $\mathrm{B}_{12}$ deficiency," JAMA, vol. 310 , no. 22 , pp. $2435-$ 2442, 2013.

[60] L. Lombardo, M. Foti, O. Ruggia, and A. Chiecchio, "Increased incidence of small intestinal bacterial overgrowth during proton pump inhibitor therapy," Clinical Gastroenterology and Hepatology, vol. 8, no. 6, pp. 504508, 2010.

[61] L. Linder, C. Tamboue, and J. N. Clements, "Drug-induced vitamin $\mathrm{B}_{12}$ deficiency: a focus on proton pump inhibitors and histamine-2 antagonists," Journal of Pharmacy Practice, vol. 30, no. 6, pp. 639-642, 2017.

[62] S. Rafiee, K. Asadollahi, G. Riazi, S. Ahmadian, and A. A. Saboury, "Vitamin B12 inhibits tau fibrillization via binding to cysteine residues of tau," ACS Chemical Neuroscience, vol. 8, no. 12, pp. 2676-2682, 2017.

[63] B. W. Penninx, J. M. Guralnik, L. Ferrucci, L. P. Fried, R. H. Allen, and S. P. Stabler, "Vitamin $B_{12}$ deficiency and depression in physically disabled older women: epidemiologic evidence from the Women's Health and Aging Study," The American Journal of Psychiatry, vol. 157, no. 5, pp. 715-721, 2000.

[64] F. Ma, T. Wu, J. Zhao et al., "Plasma homocysteine and serum folate and vitamin $B_{12}$ levels in mild cognitive impairment and Alzheimer's disease: a case-control study," Nutrients, vol. 9, no. 12, 2017.

[65] E. M. Mandelkow and E. Mandelkow, "Biochemistry and cell biology of tau protein in neurofibrillary degeneration," Cold Spring Harbor Perspectives in Medicine, vol. 2, no. 7, article a006247, 2012.

[66] T. Bottiglieri, E. Arning, B. Wasek, V. Nunbhakdi-Craig, J. M. Sontag, and E. Sontag, "Acute administration of L-DOPA induces changes in methylation metabolites, reduced protein phosphatase 2A methylation, and hyperphosphorylation of tau protein in mouse brain," The Journal of Neuroscience, vol. 32, no. 27, pp. 9173-9181, 2012.

[67] W. Wei, Y. H. Liu, C. E. Zhang et al., "Folate/vitamin-B12 prevents chronic hyperhomocysteinemia-induced tau hyperphosphorylation and memory deficits in aged rats," Journal of Alzheimer's Disease, vol. 27, no. 3, pp. 639-650, 2011.

[68] C. E. Zhang, Q. Tian, W. Wei et al., "Homocysteine induces tau phosphorylation by inactivating protein phosphatase $2 \mathrm{~A}$ in rat hippocampus," Neurobiology of Aging, vol. 29, no. 11, pp. 1654-1665, 2008.

[69] C. E. Zhang, W. Wei, Y. H. Liu et al., "Hyperhomocysteinemia increases $\beta$-amyloid by enhancing expression of $\gamma$-secretase and phosphorylation of amyloid precursor protein in rat brain," The American Journal of Pathology, vol. 174, no. 4, pp. 1481-1491, 2009.

[70] G. Ravaglia, P. Forti, F. Maioli et al., "Homocysteine and folate as risk factors for dementia and Alzheimer disease," The American Journal of Clinical Nutrition, vol. 82, no. 3, pp. 636-643, 2005.

[71] M. T. Heneka, J. J. Rodríguez, and A. Verkhratsky, "Neuroglia in neurodegeneration," Brain Research Reviews, vol. 63, no. 1-2, pp. 189-211, 2010.

[72] T. Mizuno, R. Kuno, A. Nitta et al., "Protective effects of nicergoline against neuronal cell death induced by activated microglia and astrocytes," Brain Research, vol. 1066, no. 1-2, pp. 78-85, 2005. 
[73] R. E. Gonzalez-Reyes, M. O. Nava-Mesa, K. Vargas-Sanchez, D. Ariza-Salamanca, and L. Mora-Muñoz, "Involvement of astrocytes in Alzheimer's disease from a neuroinflammatory and oxidative stress perspective," Frontiers in Molecular Neuroscience, vol. 10, p. 427, 2017.

[74] M. V. Sofroniew and H. V. Vinters, "Astrocytes: biology and pathology," Acta Neuropathologica, vol. 119, no. 1, pp. 7-35, 2010.

[75] A. A. Cronican, N. F. Fitz, T. Pham et al., "Proton pump inhibitor lansoprazole is a nuclear liver $\mathrm{X}$ receptor agonist," Biochemical Pharmacology, vol. 79, no. 9, pp. 13101316, 2010.

[76] R. Batchelor, J. F. Gilmartin, W. Kemp, I. Hopper, and D. Liew, "Dementia, cognitive impairment and proton pump inhibitor therapy: a systematic review," Journal of Gastroenterology and Hepatology, vol. 32, no. 8, pp. 1426-1435, 2017.

[77] A. M. Herghelegiu, G. I. Prada, and R. Nacu, "Prolonged use of proton pomp inhibitors and cognitive function in older adults," Farmácia, vol. 64, no. 2, pp. 262-267, 2016.

[78] M. G. Delgado, S. Calleja, L. Suarez, and J. Pascual, "Recurrent confusional episodes associated with hypomagnesaemia due to esomeprazol," BMJ Case Reports, vol. 2013, article bcr2013200501, 2013.

[79] L. Pasina, D. Zanotta, S. Puricelli, and G. Bonoldi, "Acute neurological symptoms secondary to hypomagnesemia induced by proton pump inhibitors: a case series," European Journal of Clinical Pharmacology, vol. 72, no. 5, pp. 641-643, 2016.

[80] V. S. Bebarta, J. A. King, and M. McDonough, "Proton pump inhibitor-induced rhabdomyolysis and hyponatremic delirium," The American Journal of Emergency Medicine, vol. 26, no. 4, pp. 519.e1-519.e2, 2008.

[81] S. Fujii, H. Tanimukai, and Y. Kashiwagi, "Comparison and analysis of delirium induced by histamine $\mathrm{H} 2$ receptor antagonists and proton pump inhibitors in cancer patients," Case Reports in Oncology, vol. 5, no. 2, pp. 409-412, 2012.

[82] I. Otremba, K. Wilczyński, and J. Szewieczek, "Delirium in the geriatric unit: proton-pump inhibitors and other risk factors," Clinical Interventions in Aging, vol. 11, pp. 397405, 2016.

[83] F. C. Soares, T. C. de Oliveira, L. D. de Macedo et al., "CANTAB object recognition and language tests to detect aging cognitive decline: an exploratory comparative study," Clinical Interventions in Aging, vol. 10, pp. 37-48, 2015.

[84] P. Lochhead, K. Hagan, A. D. Joshi et al., "Association between proton pump inhibitor use and cognitive function in women," Gastroenterology, vol. 153, no. 4, pp. 971979.e4, 2017.

[85] W. Li, S. Zeng, L.-S. Yu, and Q. Zhou, "Pharmacokinetic drug interaction profile of omeprazole with adverse consequences and clinical risk management," Therapeutics and Clinical Risk Management, vol. 9, pp. 259-271, 2013.

[86] C. Parsons, S. Johnston, E. Mathie et al., "Potentially inappropriate prescribing in older people with dementia in care homes," Drugs \& Aging, vol. 29, no. 2, pp. 143-155, 2012.

[87] J. Labenz, K. U. Petersen, W. Rösch, and H. R. Koelz, “A summary of Food and Drug Administration-reported adverse events and drug interactions occurring during therapy with omeprazole, lansoprazole and pantoprazole," Alimentary Pharmacology and Therapeutics, vol. 17, no. 8, pp. 1015-1019, 2003.
[88] J. Gallacher, P. Elwood, J. Pickering, A. Bayer, M. Fish, and Y. Ben-Shlomo, "Benzodiazepine use and risk of dementia: evidence from the Caerphilly Prospective Study (CaPS)," Journal of Epidemiology \& Community Health, vol. 66, no. 10 , pp. 869-873, 2012.

[89] S. Billioti de Gage, A. Pariente, and B. Begaud, "Is there really a link between benzodiazepine use and the risk of dementia?," Expert Opinion on Drug Safety, vol. 14, no. 5, pp. 733-747, 2015.

[90] M. O. Nava-Mesa, L. Jimenez-Diaz, J. Yajeya, and J. D. Navarro-Lopez, "GABAergic neurotransmission and new strategies of neuromodulation to compensate synaptic dysfunction in early stages of Alzheimer's disease," Frontiers in Cellular Neuroscience, vol. 8, p. 167, 2014.

[91] C. M. Stonnington, P. J. Snyder, J. G. Hentz, E. M. Reiman, and R. J. Caselli, "Double-blind crossover study of the cognitive effects of lorazepam in healthy apolipoprotein $\mathrm{E}$ (APOE)-ع4 carriers," The Journal of Clinical Psychiatry, vol. 70, no. 10, pp. 1379-1384, 2009.

[92] R. Gugler, M. Hartmann, J. Rudi et al., "Lack of pharmacokinetic interaction of pantoprazole with diazepam in man," British Journal of Clinical Pharmacology, vol. 42, no. 2, pp. 249-252, 1996.

[93] R. A. Lefebvre, B. Flouvat, S. Karolac-Tamisier, E. Moerman, and E. V. Ganse, "Influence of lansoprazole treatment on diazepam plasma concentrations," Clinical Pharmacology \& Therapeutics, vol. 52, no. 5, pp. 458-463, 1992.

[94] T. Ishizaki, K. Chiba, K. Manabe et al., "Comparison of the interaction potential of a new proton pump inhibitor, E3810, versus omeprazole with diazepam in extensive and poor metabolizers of $S$-mephenytoin 4'-hydroxylation," Clinical Pharmacology \& Therapeutics, vol. 58, no. 2, pp. 155-164, 1995.

[95] G. M. Pasinetti, "From epidemiology to therapeutic trials with anti-inflammatory drugs in Alzheimer's disease: the role of NSAIDs and cyclooxygenase in $\beta$-amyloidosis and clinical dementia," Journal of Alzheimer's Disease, vol. 4, no. 5, pp. 435-445, 2002.

[96] P. L. McGeer, E. McGeer, J. Rogers, and J. Sibley, "Antiinflammatory drugs and Alzheimer disease," The Lancet, vol. 335 , no. 8696 , p. 1037, 1990.

[97] A. Ghallab, "In vitro test systems and their limitations," EXCLI Journal, vol. 12, pp. 1024-1026, 2013.

[98] R. D. Bell and B. V. Zlokovic, "Neurovascular mechanisms and blood-brain barrier disorder in Alzheimer's disease," Acta Neuropathologica, vol. 118, no. 1, pp. 103-113, 2009.

[99] G. N. Shah and A. D. Mooradian, "Age-related changes in the blood-brain barrier," Experimental Gerontology, vol. 32, no. 4-5, pp. 501-519, 1997.

[100] T. O. Kleine, R. Hackler, and P. Zofel, "Age-related alterations of the blood-brain-barrier (bbb) permeability to protein molecules of different size," Zeitschrift für Gerontologie, vol. 26, no. 4, pp. 256-259, 1993. 


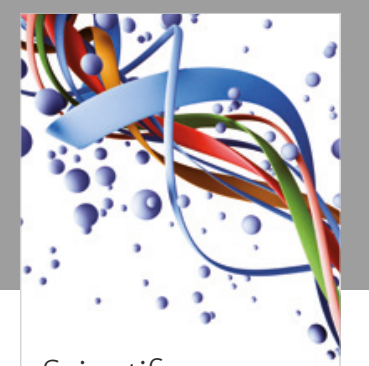

Scientifica
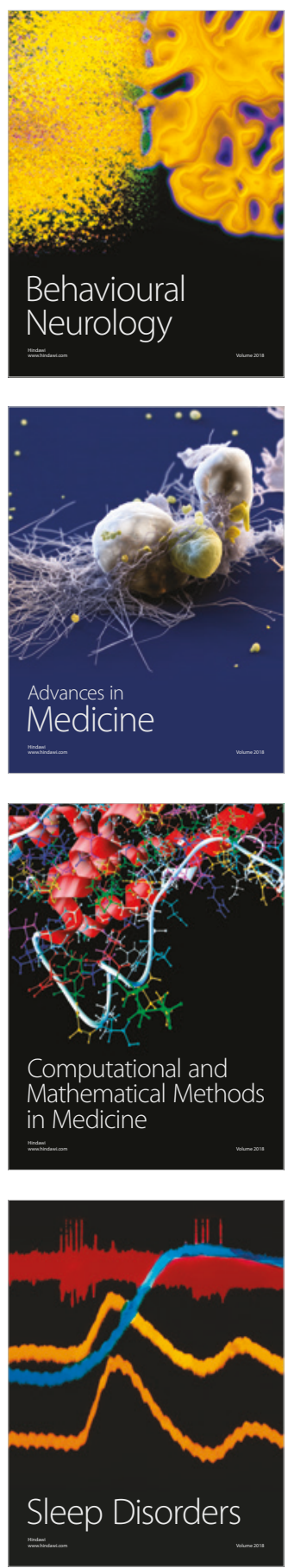

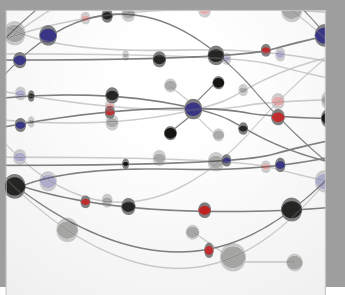

The Scientific World Journal

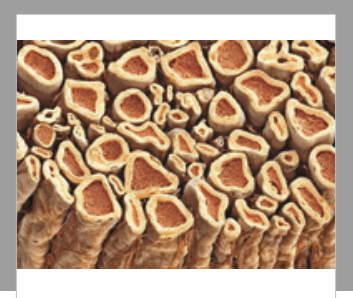

Case Reports in

Neurological Medicine

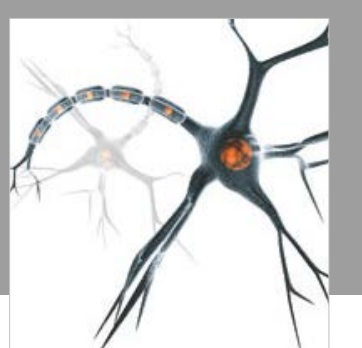

Neural Plasticity

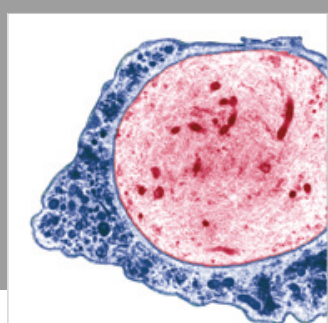

Multiple Sclerosis

International

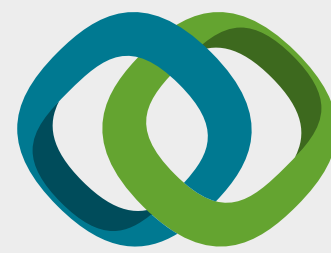

Hindawi

Submit your manuscripts at

www.hindawi.com
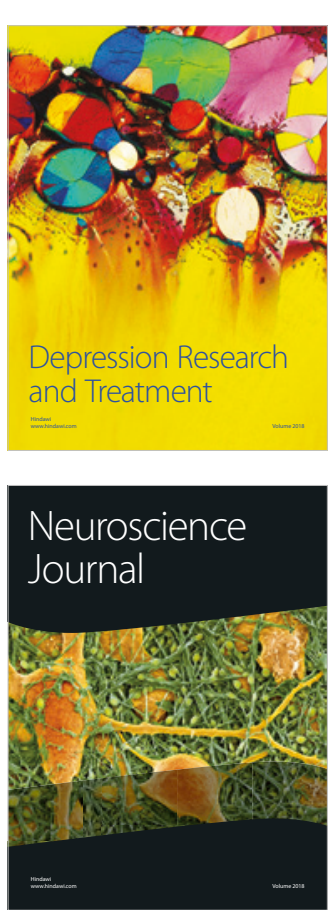

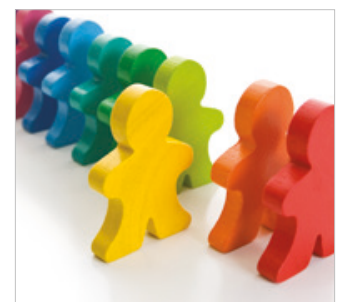

Autism

Research and Treatment
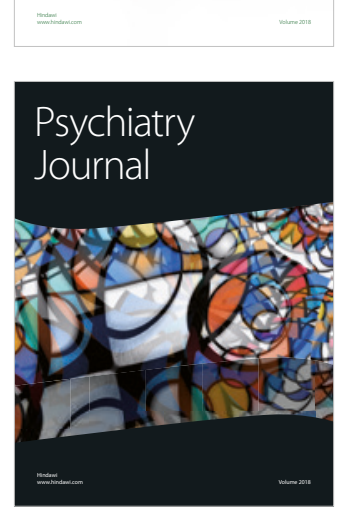
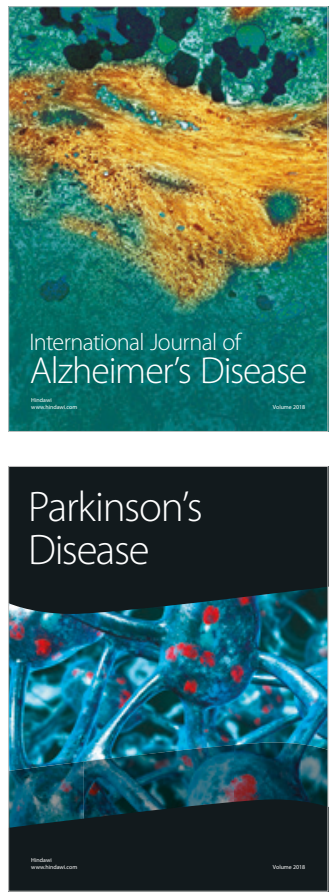
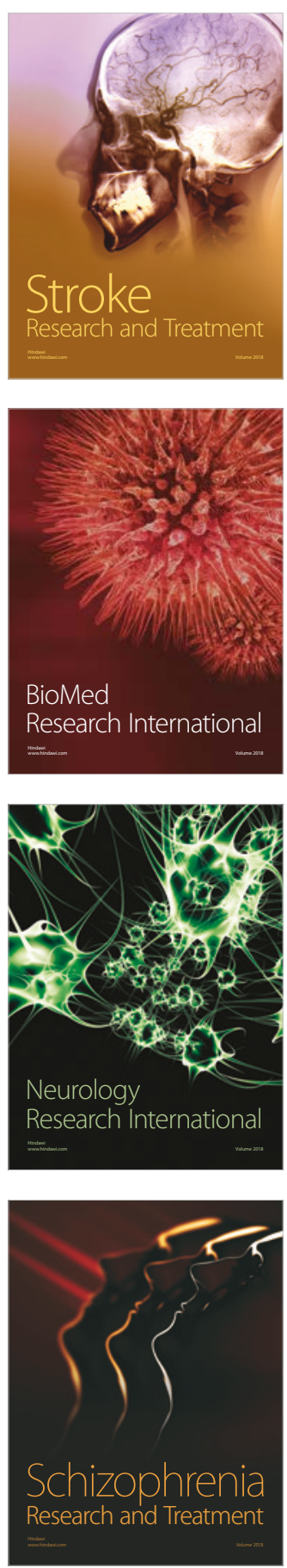